\title{
Approach of the T-CONT Allocation to Increase the Bandwidth in Passive Optical Networks
}

\author{
Zoltan BOSTERNAK, Rastislav ROKA \\ Inst. of Multimedia Information and Communication Technologies, Slovak University of Technology \\ STU FEI, Ilkovičova 3, 812 19, Bratislava, Slovakia \\ zoltan.bosternak@stuba.sk, rastislav.roka@stuba.sk \\ Submitted October 17, 2016 / Accepted April 27, 2017
}

\begin{abstract}
This paper works with the simulation of T-CONT allocation and delay analysis in passive optical networks PON. Building our networks with the PON technology we can achieve increased data rates, however we need to ensure that the idle gaps between the particular transmissions are minimal. The primary method for the upstream time slot allocation in passive optical networks is via Multi Point Control Protocol. The baseline standard of this protocol clearly defines the use of the REPORT and GATE control messages. The two optical network elements used here, the optical network unit ONT and the optical line termination OLT, located at the central office $C O$, can be scheduled to allocate the time slots. Using the control messages, a more accurate scheduling algorithm can be developed, hence we can directly improve the utilization of the bandwidth as well. In this work, we introduce the basic topology of the passive optical networks, how PON works and what basic principles of bandwidth allocation have been applied. Subsequently, we suggest a selection of methods for time slot allocation and we make an analysis on the achieved results. Our main focus is on the system load, transfer delay and the analysis of the effectivity.
\end{abstract}

\section{Keywords}

Passive optical networks, transmission delay, bandwidth allocation

\section{Introduction}

Customers or end-users in passive optical networks have an increasing demand on the size of allocated bandwidth day by day. The introduction of new services, as IPTV, HSI, video services over the Internet, increased the demands for higher bandwidth utilization in telecommunications. Demands to have as much bandwidth as possible for each customer are increasing year by year. There are different kinds of customers with various needs, therefore the size of allocated time frames as well as the transmission delays are different. Service providers have to find new solutions constantly and welcome effective ways to allo- cate time containers. The most effective solution to increase bandwidth in passive optical networks PON is to allocate time containers in a manner of different ways. With this approach, the architectures of FTTH and FTTB will be still attractive for the end users [9]. One of the important values that we need to analyze is the transmission delay that can be effectively reduced with the right way of time slot assignments. Some of the former technologies, like xDSL have similar properties though; however have restrictions with regards to the distance. Passive optical networks are available since some decades, but the important successes have been achieved with the introduction of various standards for APON, BPON, EPON and GPON [11]. The transmission medium, used in this type of networks is an optical fiber, which can have different kind of sensitivity. In passive optical network's point-to-multipoint architecture, couples of users are connected to the same optical line termination OLT via optical splitters. There are different ways of assigning the time containers. We can do it either statically or dynamically. In the oldest type of passive optical networks, the method used for bandwidth allocation was static. With statically assigned time containers in PONs, we can use time slots for every end-user, resp. optical network termination ONT separately. As soon as the facility connected to a specific ONT is not using the whole bandwidth - which has been allocated statically we can conclude the solution as non-effective. Once we choose the dynamic allocation of time containers, we are coming to the effort to fully utilize the network. With the dynamic allocation, the assignment of T-CONT is done per the requirement from the users. A well-defined algorithm of dynamic allocation is utilizing as much of the available bandwidth as can and is able to meet the requirements from the customers. Therefore, the main aim of this paper is to analyze the upstream traffic of passive optical networks, their bandwidth and the possible approaches to increase the utilization of the bandwidth as much as possible [9], [12], [15].

In the following sections, we will go thru a basic introduction of passive optical networks (Sec. 2), their functionality and properties. We will also introduce the round trip time RTT, as one of the important values with a huge impact on the network along with the MPCP protocol. In 
Sec. 3, we will name the bandwidth allocation mechanisms used in passive optical networks, whereas in Sec. 4 and 5 we will show the simulation results with the graphical interpretation of the delay analysis.

\section{Passive Optical Networks PON}

Figure 1 shows the common topology used in passive optical networks. This kind of passive optical network consists of one optical line termination OLT at the central office $\mathrm{CO}$. On the other hand, there are several end-users with optical network termination/unit connected to it via optical splitter. The optical line termination OLT is the equipment responsible to provide different kinds of services for several users. Users are easily connected to the optical network units. Between OLT and each ONT there are different values of round trip time RTT. This is the time that takes for the optical signal to be sent with an additional time of acknowledgment what is basically some kind of confirmation that the signal was successfully received [1], [2], [13], [14], [16].

There are two ways of data transmission in PON, the downstream and the upstream direction. The optical splitter has the possibility to broadcast the signal in downstream direction to every ONT, whereas in the upstream direction there is no direct connection between optical network units. It means that the upstream direction can transfer the data only to the optical line termination OLT. In the downstream direction the PON acts as a point-to-multipoint P2MP and in the reverse upstream direction we call it multipoint-to-point solution.

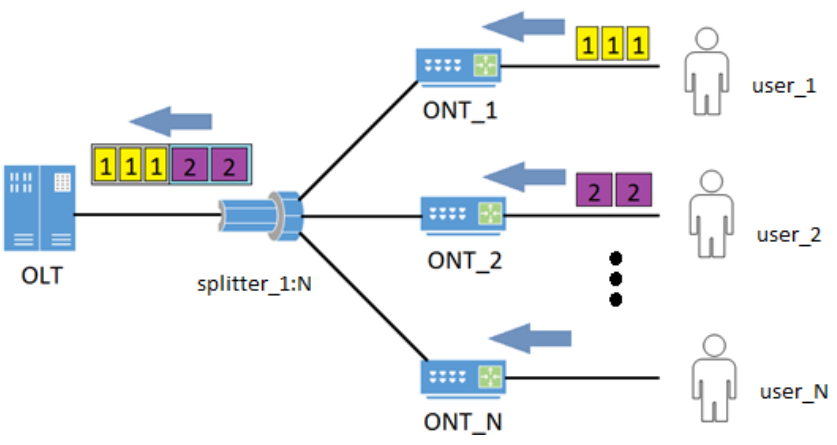

Fig. 1. The common topology in PON for the upstream traffic.

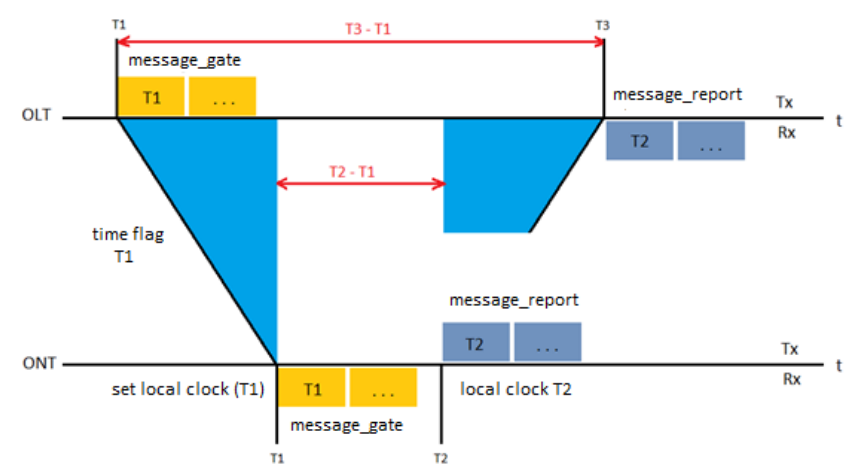

Fig. 2. Illustration of a round trip time RTT.

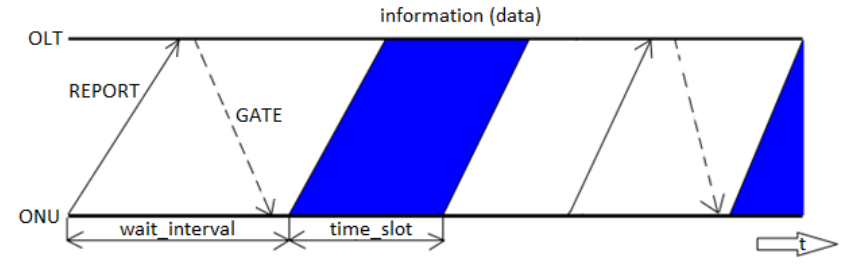

Fig. 3. REPORT/GATE messages in the MPCP mechanism.

The transfer in downstream direction is done with one wavelength. The entities used in the upstream direction are called time slots (containers). Only one unique optical network unit can transfer data within a specific allocated time slot [15], [16]. That's the way how passive optical networks prevent collisions to happen. The unit ONT gathers and buffers the data to transfer over the fiber unless there is a valid time slot allocated for it. The most recent bandwidths are associated with different types of PON. The Ethernet PON is using $1.25 \mathrm{~Gb} / \mathrm{s}$ in both upstream/ downstream directions, whereas in Gigabit capable PON it can be either symmetric $(1.24416 \mathrm{~Gb} / \mathrm{s} ; 1.24416 \mathrm{~Gb} / \mathrm{s})$ or asymmetric $(1.24416 \mathrm{~Gb} / \mathrm{s} ; 2.48832 \mathrm{~Gb} / \mathrm{s})$. We need to consider the $8 \mathrm{~B} / 10 \mathrm{~B}$ line coding, so in case of EPON the real transmission capacity will be $1 \mathrm{~Gb} / \mathrm{s}$. For the bandwidth allocation in EPON, the mechanism used is MPCP with the help of GATE/REPORT messages [12].

\section{Bandwidth Allocation Methods}

Previous generations of passive optical networks are allocating time slots statically. Each end-user can receive a pre-defined statically assigned time slot regardless their use. It can be beneficial in case of using a VoIP or TDM, where the network has the demand for permanent and statically assigned bandwidth. However we need to be aware of the fact, that the unused time slot by the current ONT cannot be used by other ONTs. It can be a good solution though, when the requirement for the bandwidth is constant. As soon as the ONT is idle, it leads to a non-effective solution that cannot be acceptable anymore. The root of the bandwidth allocation in PON is called IPACT. This is the method of bandwidth assignment with statistical multiplexing, used in several EPON solutions and we can refer to it in case of further development. With ensuring the quality of services QoS we are approaching to the solutions as DEB, BGP (absolute) and DBA for multimedia (relative). We call them the basic taxonomy of bandwidth assignments. So let's focus on Interleaved Polling with Adaptive Cycle Time IPACT. We have four major types of assignments [1], [12].

- The first one is the Fixed Bandwidth Allocation FBA. With this method we are allocating a fix time container for each optical network unit. In this way, an exact grant cycle is assigned and during that time window the specific ONT can send its buffered data. In this allocation method - as we can see from the name convention - a fixed bandwidth is allocated for every unit and is acting as a time division multiplex- 
ing TDM. This is the simplest way of assigning a unique time window.

- There is also an alternative of this first method, which is named as Limited Bandwidth Allocation LBA. The mechanism used here is called REPORT/GATE. Bandwidth allocation method LBA is monitoring the incoming traffic based on this mechanism. The time window that can be allocated to every ONT is limited with a maximum value of possible time slot. The optical network unit sends a report to the optical line termination informing its own front resp. buffer-size. If the informed size of time window is lower than the value set as a maximum limit, it will assign the requested value to that ONT. Otherwise it will grant only the maximum possible value within the network topology, so the maximum possible value per the ONT.

- Another form of bandwidth allocation can be based on a credit assigned to the queue. The credit associated with the Credit Based Allocation CBA can be either constant or linear. The result of the bandwidth assignment allocated for each ONT depends on the requested bandwidth per the associated buffer where the traffic is waiting for transfer plus the additional credit - which is constant or linear.

- The last option is the method named as Excessive Bandwidth Allocation EBA. In passive optical networks it sometimes happens, that there is an unused bandwidth. The sum of these unused bandwidths in lowly utilized ONTs is called excessive bandwidth which we can further use among other ONTs. This is an important mile in moving forward toward the effective utilization in passive optical network to achieve better performance and provide better services for end-users [1], [12-16].

\section{Simulation of T-CONT Allocation}

For the simulation of the upstream traffic in passive optical networks, their bandwidth assignments and calculation of their effectivity we can use different ways. We may have different programming languages, like $\mathrm{C} / \mathrm{C}++$, Matlab etc. We have decided to use a programming language developed by MathWorks. Matlab allows matrix manipulations, plotting of functions and data, implementation of algorithms where possible, creation of various interfaces, and also it can interface with programs written in other languages, including $\mathrm{C}, \mathrm{C}++$, Java, Fortran and Python [17], ]18]. With regards to the simulation, our initial detail used by the calculation is the round trip time, which is the delay of the optical signal, necessary to ensure data traffic without any collision - as we already know the synchronization has to be done between units OLT and ONT with a local clocks and the GATE message is responsible to deliver this information to ONTs. In our simulation, we suggest several methods for time slot assigning in case of upstream direction for passive optical networks. These methods can be easily compared with another already existing ones (e.g. Fixed Bandwidth Allocation, FBA, or Limited Bandwidth Allocation, LBA), so we can decide on their effectivity of use. There are different kinds of topologies that can be used for the simulation, like ring, star and bus. The common topology in passive optical networks is the star topology. Right before we start the simulation, we need to consider some initial parameters in PON. In our simulation - for easier case study - we will consider the transfer delay only, which basically depends on the physical length of the optical fiber. In our case, we use four different units ONT - each with different distance from OLT - interconnected via optical splitter. Mechanism of MPCP is using a technique similar to TDM, therefore the decision of which ONT and what time will send its data depends on OLT. In the calculation process time interval $\mathrm{T}_{\mathrm{PR}}$, OLT makes its decision - certainly within one polling cycle - only after gathering all the necessary information from the REPORT message. When the time windows are calculated, OLT transmits the GATE message based on the appropriate methods of bandwidth assignment. In this work the main focus is on the transmission delay in PON [17].

The upstream bandwidth - shown in Fig. 1 (from each ONT towards the OLT unit) - is the theoretical bandwidth given by the specification for Ethernet PON. In some cases, SLA has to be specified in the network topology so we can ensure that specific ONTs are not disadvantaged. Hence, the following has to be defined:

$$
B W_{i \mathrm{SLA}}=\frac{B W U_{\mathrm{FULL}}}{N}[\mathrm{Mbps}]
$$

where $i=1,2, \ldots, N, B W_{i \mathrm{SLA}}$ is the bandwidth that can be assigned for each optical network unit, $B W U_{\mathrm{FULL}}$ is the upstream bandwidth and $N$ is the number of ONTs in the topology. The maximum distance $L_{\mathrm{M}}$, defined between OLT and several optical network units is defined in the recommendation ITU-T G.983. The sum of each bandwidth $B W R E Q_{\mathrm{ONU} i}$ that can be granted for each ONT separately cannot exceed the whole upstream bandwidth of the system PON.

$$
B W R E Q_{\mathrm{ONU}_{-} 1}+\ldots+B W R E Q_{\mathrm{ONU}_{-} N} \leq B W U_{\mathrm{FULL}} .
$$

Another important value is the guard time $T_{\mathrm{GU}}$. In fact, this time is used for the compensation of the laser on/off time $T_{\mathrm{LOO}}$ and the delay caused by round trip time $R T T$. With regards to the guard time, the common time used can be between 50 and $150 \mu \mathrm{s}$. We've chosen to use the guard time $T_{\mathrm{GU}}$ of $120 \mu$ s to see what happens in case of a middle value. Certainly in future researches, we will use a modification of this time and analyze the difference between each value. In the simulation we consider the case when every unit ONT sends its bandwidth request to the OLT in the same time - so all ONTs want to transfer data synchronously. Each packet is transmitted concurrently, affiliated with the control messages that are responsible to oversee the whole transfer [17], [18]. 


\begin{tabular}{|c|c|c|}
\hline Number of ONTs & $N$ & $4 / 8$ \\
\hline Upstream bandwidth & $B W U_{\mathrm{FULL}}$ & $\begin{array}{c}1.25 \mathrm{~Gb} / \mathrm{s} \\
(\text { after } 8 / 10 \mathrm{~B} \text { line coding } 1 \mathrm{~Gb} / \mathrm{s})\end{array}$ \\
\hline Max. distance & $L_{\mathrm{M}}$ & $20 \mathrm{~km}$ \\
\hline Guard time & $T_{\mathrm{GU}}$ & $50-150 \mu \mathrm{s}$ \\
\hline Laser on-off & $T_{\mathrm{LOO}}$ & $512 \mathrm{~ns}$ \\
\hline Request processing & $T_{\mathrm{PR}}$ & $15 \mu \mathrm{s}$ \\
\hline
\end{tabular}

Tab. 1. The initial configuration of the simulated environment.

The following methods have been defined in this paper:

- The first method - the method of static assignments is the default method of allocation bandwidth among different users with various requirements. The time slots in method 1 are allocated constantly without considering any requirements. This is the initial method of the well-known Fixed Bandwidth Allocation FBA. The main disadvantage of this method is that the provided bandwidth cannot be efficiently utilized as the particular slots are constantly allocated whether they are needed or not.

- In passive optical networks, each optical network unit ONT has its own unique identification number ONT ID. It is the number of the ONT which is initiating the traffic. In case of no number for the ONT, it needs to be configured with a value of ' 255 ' - which is indicating the ONT without a proper value. In the downstream direction, the field used for identifying the proper T-CONT is called AID. Optical line termination compares this field of AID with the ONT ID. In case of match we know that the correct ONT is sending the data. Therefore we have chosen to assign T-CONTs based on the ONT ID - which is the second method.

- As we already know, another important value in passive optical networks is the transfer delay. The value of delay also depends on other values, like the length of cable we use. Attenuation or signal loss in fiber and also in the optical splitter can be another way to have the requested bandwidth to be assigned. If we come to the end, we can say that assigning time containers based on a delay in the network probably won't be so efficient and it is not so stable. To sort the incoming requests based on the queue, we have used a part of the source code shown in Tab. 2. As the time containers will be assigned per the size of the delay, their characteristic will be linear though, but unevenly distributed - we will use it as method 3 .

- As the time window assigned for each bandwidth request is a value of requested bandwidth divided with the capacity of the link, we can conclude that assigning the bandwidth based on the requested values would be also a good option - method 4 . We can allocate the bandwidth from the ONT which is requesting the higher bandwidth towards the lower ones or vice versa.

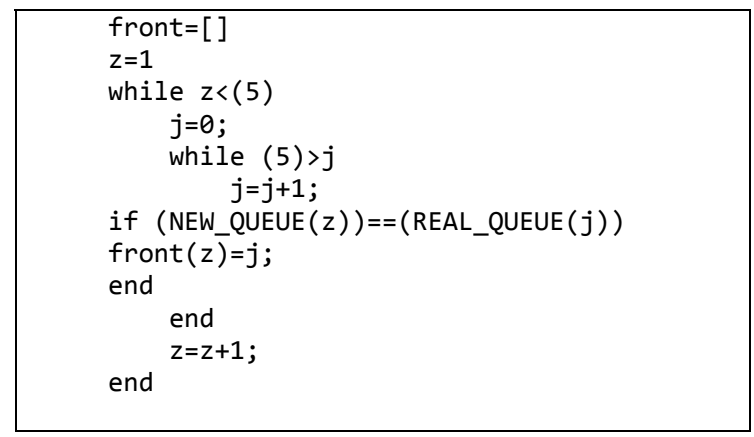

Tab. 2. The condition based while cycle to sort the front of requests.

Following the protocol, used for the bandwidth allocation (Fig. 3), we can conclude that with time slot allocation per the above suggested methods we will receive various results. This can be explained with the response time for the value, that we are monitoring (transmission delay, bandwidth requests, ONT ID, etc.). Obviously, the most wrongly utilized bandwidth result can be expected with our first method, as the bandwidth is assigned statically regardless our needs. We can easily improve the bandwidth utilization with assigning the time slot per our requirements, therefore the gaps between the specific transmissions are minimized.

All the above ways of assigning the T-CONTs are theoretical modifications of the previous well-known methods and could be used to compare the efficiency in the analysis of bandwidth allocation.

\section{Results of T-CONT Allocation}

In the simulation, we have used four optical network units. At this time, we do not consider any physical layer information; however we need to know that the round trip time is highly dependent on the distance between OLT and several ONT units. To illustrate the difference between various bandwidth allocation mechanisms, we introduced four different network units ONT, each with different value of RTT. As the services that the customers can use behind each optical network unit may differ, therefore we have also chosen different values of bandwidth requests associated with specific network units in our simulation.

As previously defined in Sec. 3, where the bandwidth allocation methods have been discussed, we will use four different methods in the simulation. The first method is used in case of fixed bandwidth allocation FBA. In this case, we do not even need to have bandwidth requests from the network units. Whereas each optical network unit requests the necessary bandwidth allocation slot for the transfer, the optical line termination OLT will grant the time slot statically regardless their needs.

The following three methods (method 2, 3 and 4) can be used for the comparison with the well-known FBA method. As we can see, method 2 and method 3 are more effective ways of assigning time slots, however an algo- 
rithm is needed to prioritize and sort the ONTs per the requested values. The most effective solution would be to assign bandwidth in different ways based on the size of the requested time slots.

In Figure 4 we can observe that four different methods (1-4) achieving different results with regards to the effectivity. In the last diagram 'Average Delay' (Fig. 5) we can notice, that our most effective way is the time slot assignment based on the bandwidth requested per each customer depending on the service they use.

Now let's take a look on a simulated environment with an increased number of optical network units. We will keep the four methods used for the time slot allocation. As we can see in Tab. 5, the bandwidth utilization is increased with $12.88 \%$.

Simulation results for this scenario show that the system delay will change as seen in Tab. 6 .

With the increasing amount of optical units in the network, the users can eventually request fewer amounts of data for the upstream traffic. More users in the network topology mean a slight increase of the average system delay, too (Fig. 5).

\begin{tabular}{|c|c|c|}
\hline ONT ID & Bandwidth request & $\boldsymbol{R T T}$ \\
\hline ONT_1 & $87 \mathrm{Mbit} / \mathrm{s}$ & $67 \mu \mathrm{s}$ \\
\hline ONT_2 & $204 \mathrm{Mbit} / \mathrm{s}$ & $102 \mu \mathrm{s}$ \\
\hline ONT_3 & $162 \mathrm{Mbit} / \mathrm{s}$ & $34 \mu \mathrm{s}$ \\
\hline ONT_4 & $124 \mathrm{Mbit} / \mathrm{s}$ & $92 \mu \mathrm{s}$ \\
\hline Total bandwidth: & $\mathbf{5 7 7} \mathbf{M b i t} / \mathbf{s}$ & - \\
\hline Utilization: & $\mathbf{5 6 . 2 9} \%$ & - \\
\hline
\end{tabular}

Tab. 3. The basic parameters for the simulation.

\begin{tabular}{|c|c|c|c|c|}
\hline METHOD & no_1 & no_2 & no_3 & no_4 \\
\hline ONT_1 $1_{\text {TIME }}(\mu \mathrm{s})$ & 0 & 0 & 162.0705 & 0 \\
\hline ONT_2 $2_{\text {TIME }}(\mu \mathrm{s})$ & 250.0025 & 87.0025 & 373.1105 & 373.0855 \\
\hline ONT_3 $3_{\text {TIME }}(\mu \mathrm{s})$ & 500.0730 & 291.0730 & 0 & 211.0830 \\
\hline ONT_4 4 TIME $(\mu \mathrm{s})$ & 750.0855 & 453.0855 & 249.0730 & 87.0125 \\
\hline Average Delay $(\mu \mathrm{s})$ & 375.0402 & 207.7902 & 196.0635 & 167.7952 \\
\hline
\end{tabular}

Tab. 4. The results of the simulation.

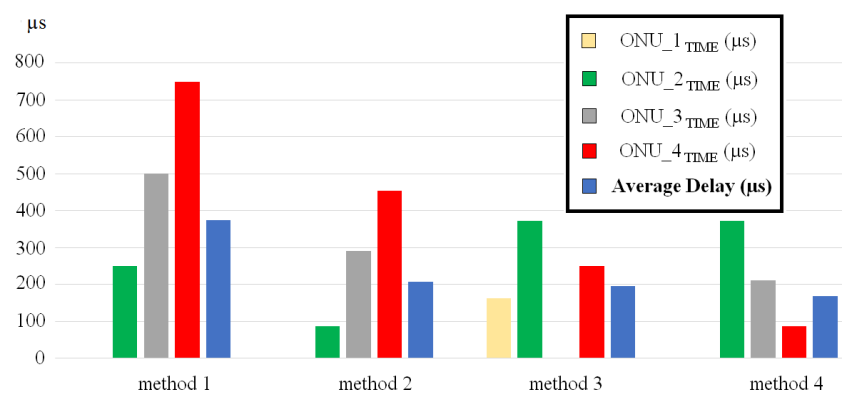

Fig. 4. The comparison of the results for methods 1-4.

\begin{tabular}{|c|c|c|}
\hline ONT ID & Bandwidth request & $\boldsymbol{R T T}$ \\
\hline ONT_1 & $91 \mathrm{Mbit} / \mathrm{s}$ & $71 \mu \mathrm{s}$ \\
\hline ONT_2 & $117 \mathrm{Mbit} / \mathrm{s}$ & $104 \mu \mathrm{s}$ \\
\hline ONT_3 & $67 \mathrm{Mbit} / \mathrm{s}$ & $48 \mu \mathrm{s}$ \\
\hline ONT_4 & $102 \mathrm{Mbit} / \mathrm{s}$ & $69 \mu \mathrm{s}$ \\
\hline ONT_5 & $45 \mathrm{Mbit} / \mathrm{s}$ & $99 \mu \mathrm{s}$ \\
\hline ONT_6 & $93 \mathrm{Mbit} / \mathrm{s}$ & $38 \mu \mathrm{s}$ \\
\hline ONT_7 & $106 \mathrm{Mbit} / \mathrm{s}$ & $86 \mu \mathrm{s}$ \\
\hline ONT_8 & $88 \mathrm{Mbit} / \mathrm{s}$ & $108 \mu \mathrm{s}$ \\
\hline Total bandwidth: & $\mathbf{7 0 9} \mathbf{M b i t} / \mathbf{s}$ & - \\
\hline Utilization: & $\mathbf{6 9 . 1 7} \%$ & - \\
\hline
\end{tabular}

Tab. 5. The upgraded parameters for the simulation.

\begin{tabular}{|c|c|c|c|c|}
\hline METHOD & no_1 & no_2 & no_3 & no_4 \\
\hline ONT_1 1 TIME $(\mu \mathrm{s})$ & 0 & 0 & 262.1355 & 200.0865 \\
\hline ONT_2 $2_{\text {TIME }}(\mu \mathrm{s})$ & 125.0050 & 91.0005 & 504.2109 & 592.2074 \\
\hline ONT_3 3 TIME $(\mu \mathrm{s})$ & 250.0570 & 208.0570 & 93.0355 & 45.0565 \\
\hline ONT_4 $4_{\text {TIME }}(\mu \mathrm{s})$ & 375.0925 & 275.0925 & 160.0690 & 384.1884 \\
\hline ONT_5 $5_{\text {TIME }}(\mu \mathrm{s})$ & 500.0980 & 377.0980 & 459.1544 & 0 \\
\hline ONT_6 $6_{\text {TIME }}(\mu \mathrm{s})$ & 625.1644 & 422.1644 & 0 & 291.1530 \\
\hline ONT_ 7 TIME $(\mu \mathrm{s})$ & 750.1829 & 515.1829 & 353.1360 & 486.2069 \\
\hline ONT_ $8_{\text {TIME }}(\mu \mathrm{s})$ & 975.1794 & 621.1794 & 621.2074 & 112.0530 \\
\hline Average Delay $(\mu \mathrm{s})$ & 437.5968 & 313.7218 & 306.6186 & 263.8690 \\
\hline
\end{tabular}

Tab. 6. The results of simulation no.2.

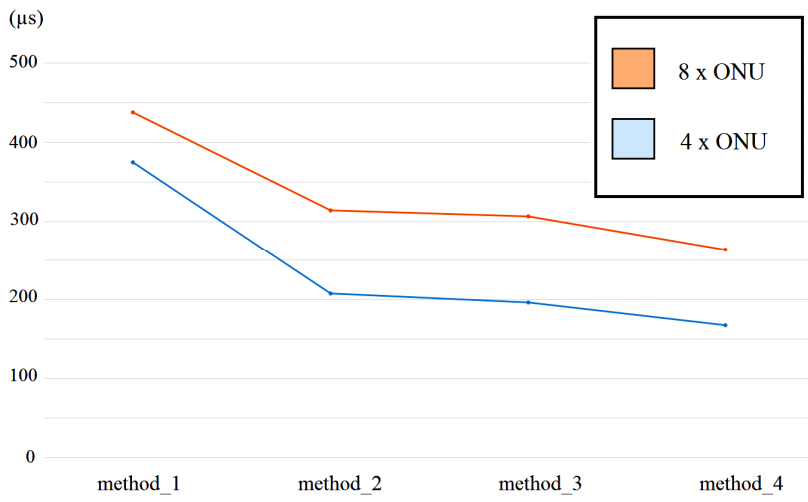

Fig. 5. System Delay with an increasing number of ONUs.

\section{Conclusions}

In this paper, our main focus was on the T-CONT allocation in the upstream direction for passive optical networks. We have introduced the round trip time $R T T$, as one of the important values in PON. Going forward, the MPCP protocol has been described. We have provided a slight overview of the bandwidth allocation mechanisms used in passive optical networks. The simulation results have been shared as well, with a graphical interpretation of the delay analysis. A simulation has been made for specific amount 
of optical network units in the area of passive optical networks. As we might expect, the results can differ with increasing number of optical network units what the results from Tab. 6 also prove. The presented results represent requests with randomly entered requirements. Hence, in future works and researches the main focus will be to increase the number of ONTs up to 128, modification of the transmission capacity and adjusting the guard time in passive optical networks. Nevertheless, we need to consider the utilization of the bandwidth as well, so we can have some real information on how the less and more utilized network topology will look like. With an analysis from less utilized network up to a fully utilized network we can see more detailed information on how efficient the suggested algorithm can be in the real world.

\section{Acknowledgements}

This work is a part of research activities conducted at the Slovak University of Technology Bratislava, Inst. of MICT, within the KEGA agency project - 007STU-4/2016 "Progressive educational methods in the field of telecommunications multiservice networks" and VEGA agency project - 1/0462/17 "Modeling of qualitative parameters in IMS networks".

\section{References}

[1] MCGARRY, M. P., MAIER, M., REISSLEIN, M. Ethernet PONs: A survey of Dynamic Bandwidth Allocation (DBA) algorithms. IEEE Communications Magazine, 2004, vol. 42, no. 8, p. 9-14

[2] HOOD, D., TROJER, E. Gigabit-capable Passive Optical Networks. Wiley, 2012. ISBN: 978-0-470-93687-0

[3] KRAMER, G., MUKHERJEE, B., PESAVENTO, D. G. IPACT: A dynamic protocol for an Ethernet PON (EPON). IEEE Communications Magazine, 2002, vol. 40, no. 2, p. 74-80.

[4] CommScope Solutions Marketing (White Paper). GPON \& EPON Comparison, 8 pages, 2013, Available at:

http://www.commscope.com/Docs/GPON_EPON_Comparison_W P-107286.pdf

[5] LUO, Y., ANSARI, N. Bandwidth allocation for multiservice access on EPONs. IEEE Communications Magazine, 2005, vol. 43, no. 2, p. S16-S21. DOI: 10.1109/MCOM.2005.1391498

[6] BYUN, H. J., NHO, J. M., LIM, J. T. Dynamic bandwidth allocation algorithm in Ethernet passive optical networks. Electronics Letters, 2003, vol. 39, no. 13, p. 1001-1002. DOI: 10.1049/el:20030635

[7] MA, M., ZHU, Y., CHENG, T. H. A bandwidth guaranteed polling MAC protocol for Ethernet passive optical networks. In The $22^{\text {nd }}$ Annual Joint Conference of the IEEE Computer and Communications Societies INFOCOM. San Francisco (CA, USA), 2003, vol. 1, p. 22-31. DOI: 10.1109/INFCOM.2003.1208655

[8] ITU-T G.983.1: Broadband Optical Access Systems Based on Passive Optical Networks (PON). Available at: http://www.itu.int/rec/T-REC-G.983.1/en
[9] OZIMKIEWICZ, J., RUEPP, S., DITTMANN, L., et al. Dynamic bandwidth allocation in GPON networks. In Recent Advances in Circuits, Systems, Signal and Telecommunications (Proceedings of the 4th WSEAS International Conference). Cambridge (USA), 2010, p. 182-187. ISBN: 978-960-474-152-6, ISSN: 1790-5117

[10] CHOI, S. I., HUH, J. D. Dynamic bandwidth allocation algorithm for multimedia services over Ethernet PONs. ETRI Journal, 2002, vol. 24 , no. 6 , p. 465-468. DOI: 10.4218/etrij.02.0202.0002

[11] CALE, I., SALIHOVIC, A., IVEKOVIC, M. Gigabit Passive Optical Network - GPON. In 29th IEEE International Conference on Information Technology Interfaces (ITI 2007). Cavtat (Croatia), 2007, p. 679-684. DOI: 10.1109/ITI.2007.4283853

[12] YAKHLEF, S. K., ABUSAEEDA, O., SHERINA, I. A comparison of dynamic bandwidth allocation and costs for EPON and GPON technologies. International Journal of Engineering and Innovative Technology, 2015, vol. 4, no. 7, p. 31-35. ISSN: 2277-3754

[13] RADZI, N. A. M, DIN, N. M., SADON, S. K., et al. Dynamic bandwidth allocation EPON survey. In IEEE Student Conference on Research and Development (SCOReD). Putrajaya (Malaysia), 2013, p. 149-153. DOI: 10.1109/SCOReD.2013.7002561

[14] QUI, I. A dynamic bandwidth allocation algorithm in EPON networks. Journal of Theoretical and Applied Information Technology, 2012, vol. 44, no. 2, p. 278-282. ISSN: 1992-8645

[15] ROKA, R. Hybrid PON Networks - Features, Architectures and Configuration. Saarbrücken (Germany): LAP Lambert Academic Publishing, 2015. ISBN: 978-3-659-43686-4

[16] LAM, C. Passive Optical Networks - Principles and Practice. San Diego (USA): Academic Press Elsevier, 2007. ISBN 978-0-12373853-0

[17] ROKA, R., CERTIK, F. Simulation Tools in Broadband Passive Optical Networks. Chapter in Simulation Technologies in Networking and Communications: Selecting the Best Tool for the Test. Pathan, A., Monowar, M., S. Khan, S. (eds.). CRC Press, 2014 , p. $337-364$. DOI: $10.1201 / \mathrm{b} 17650-16$

[18] KLUCIK, S., CHROMY, E., BARONAK, I. Model to increase the number of output sates of a random variable using a histogram based PDF. Wireless Personal Communications, 2015, vol. 85, no. 1 , p. 137-149. DOI: $10.1007 / \mathrm{s} 11277-015-2731-5$

\section{About the Authors...}

Zoltán BOSTERNÁK was born in Nové Zámky, Slovakia in 1988. He received his B.Sc and M.Sc. in Electrical Engineering at the Dept. of Telecommunications, Slovak University of Technology, in 2010 and 2012. Since 2013 he had been working as a Network Engineer at AT\&T, subsequently since 2015 as a Network Implementation Engineer at Hewlett-Packard Enterprise. Now, his function is a Senior Network Optimizations Engineer at Dell Technologies, where he is working on optimization and development of network management platforms of the internal Dell international network. His research interests include networking, bandwidth allocation, passive optical networks and their effectivity of use.

Rastislav RÓKA was born in Šal'a, Slovakia, in 1972. He received his M.Sc. and Ph.D. degrees in Telecommunications from the Slovak University of Technology, Bratislava, in 1995 and 2002. Since 1997, he has been working as a senior lecturer at the Inst. of Telecommunications, 
Faculty of Electrical Engineering and Information Technology, Slovak University of Technology in Bratislava. Since 2009, he is working as an associated professor at this institute. His research and educational activities are focused on the signal transmission realized in fixed transmis- sion media, optocommunication transmission systems and networks. A main effort is dedicated to effective utilization of the optical fiber's transmission capacity by using various optical signal processing techniques and dynamic bandwidth and wavelength allocation algorithms. 